collected as independent variables. Chi-square Automatic Interaction Detector (CHAID) method was used to examine associations between independent variables and item scores of the BI.

Results: Muscle strength and history of stroke were parent node and child node for most of BADL limitations, respectively. Upper limb muscle strength $(\leq 3)$ was a major predictor for dependence in feeding, grooming, toileting, dressing, and transfer, while lower limb muscle strength $(\leq 3)$ was a major predictor for limitation in mobility.

Conclusions: Muscle strength was the strongest predictor of BADLs among people with severe disability. Muscle strength grading may be optimal for designing supporting strategies for people with severe disabilities.

Keywords: Disability, Basic activities of daily living, Long-term care, CHAID analysis

\title{
409 - Psychometric features of Comprehensive Geriatric Assessments (CGAs) in long-term and community care settings: A Systematic Review
}

Author List

Mauricio Molinari Ulate ${ }^{1,2}$, Aysan Mahmoudi ${ }^{1,2}$, Henriëtte G. van der Roest ${ }^{5}$, and Manuel A. Franco-Martín ${ }^{1,3,4}$

${ }^{1}$ Institute of Biomedical Research of Salamanca, University of Salamanca

${ }^{2}$ Department of Research and Development, INTRAS Foundation

${ }^{3}$ Psychiatric Department, Zamora Healthcare Complex

${ }^{4}$ Psychiatric Department, Hortega University Hospital

${ }^{5}$ Department on Aging, Netherlands Institute of Mental Health and Addiction (Trimbos Institute)

Background:

Comprehensive Geriatric Assessments (CGAs) incorporate the key principles of integrated care delivery, which is regarded as the most effective approach of care provisioning to face challenges of dementia care, such as uncoordinated care, risk of hospitalizations, care planning and unmet needs. For this reason, implementing CGAs in care practice might be highly relevant, however, to do so, we must consider their psychometric characteristics.

Research Objective:

To provide insight into the content and the reliability and validity of CGAs used in long-term care and home care settings.

Method:

A search strategy was conducted in PubMed, CINAHL, and Web of Science, targeting studies that have focused on the validity and reliability of available CGAs for long-term and home care settings. Studies in English and Spanish and published up to July 13, 2021, were considered.

Preliminary results of the ongoing study:

A total of 72 studies reporting on the validity/reliability of 13 different CGAs were identified. For long-term care facilities, five CGAs were reported; for home care, eight assessment tools were targeted. Most of the CGAs covered a wide range of domains, such as Physical Health, Functional, Mental Health, and Social Status. Evidence for good to excellent validity and reliability was reported for various instruments.

Conclusion: Resident Assessment Instrument-Minimum Data Set (RAI-MDS) and subsequent updated instruments reported strong evidence of good to excellent validity and reliability for multiple countries. For this reason, interRAI LTCF and interRAI HC are recommended to be use for long-term and home care 
facilities, as they are the latest versions of the RAI-MDS.

This project is part of the Marie Skłodowska Curie Actions Innovative Training Network H2020-MSCA-ITN, under grant agreement number 813196

\section{0 - A Systematic Review on Digital Health Interventions (DHIs) supporting the administration of Comprehensive Geriatric Assessments (CGAs) use in long-term and home care settings}

Author List

Mauricio Molinari Ulate ${ }^{1,2}$, Aysan Mahmoudi ${ }^{1,2}$, Esther Parra-Vidales ${ }^{2}$, Juan-Luis Muñoz-Sánchez ${ }^{4}$, Henriëtte G. van der Roest ${ }^{5}$, and Manuel A.Franco-Martín ${ }^{1,3,4}$

${ }^{1}$ Institute of Biomedical Research of Salamanca, University of Salamanca

${ }^{2}$ Department of Research and Development, INTRAS Foundation

${ }^{3}$ Psychiatric Department, Zamora Healthcare Complex

${ }^{4}$ Psychiatric Department, Hortega University Hospital

${ }^{5}$ Department on Aging, Netherlands Institute of Mental Health and Addiction (Trimbos Institute)

Background:

Health systems are in the need for novel approaches to tackle the challenges from the demographic transition to ageing populations. An effective approach to face these challenges is integrated care delivery. Implementing Comprehensive Geriatric Assessments (CGAs) in Health Ageing might be highly relevant to incorporate this approach. However, this implementation should be supported by Digital Health Interventions (DHIs) in order to reach their full capacity.

Research Objective:

To identify the DHIs facilitating the administration of CGAs used in long-term care and home care settings and provide an insight on their characteristics and stage of maturity and evaluation.

Method:

A search strategy was conducted in PubMed, CINAHL, and Web of Science, targeting studies evaluating the DHIs facilitating the administration of CGAs used in long-term care and home care settings. Studies in English and Spanish and published up to July 26, 2021 were considered.

Preliminary results of the ongoing study:

A total of four papers describing three digital platforms supporting the administration of the CGAs were identified. Information on implementation reported less completion of some sections affecting the CGAs outcomes, assessments mostly being the responsibility of nurses, and missing data related with less quality of care. Limitations and barriers regarding their usability and feasibility were also identified.

Conclusion:

The inclusion of safe data storage, automatic notifications for assessment completion, automatic calculation of final outcomes, and facilitation of multidisciplinary assessments, were identified as key features to enhance the implementation of digital platforms facilitating the administration of CGAs. Nonetheless, information regarding technical features and hardware information of the digital platforms was scarce.

This project is part of the Marie Skłodowska Curie Actions Innovative Training Network H2020-MSCA-ITN, under grant agreement number 813196 